\title{
Through-thickness stress relaxation in bacterial cellulose hydrogel
}

\author{
Xing Gao ${ }^{1}$, Piotr Kuśmierczyk ${ }^{2}$, Zhijun Shi ${ }^{3}$, Changqing Liu ${ }^{1}$, Guang Yang ${ }^{3}$, Igor \\ Sevostianov $^{4}$ and Vadim V Silberschmidt ${ }^{11}$
}

${ }^{1}$ Wolfson School of Mechanical and Manufacturing Engineering, Loughborough University, Loughborough, UK

${ }^{2}$ Department of Mathematics, Aberystwyth University, Aberystwyth, UK

${ }^{3}$ College of Life Science and Technology, Huazhong University of Science and Technology, Wuhan, China

${ }^{4}$ Department of Mechanical and Aerospace Engineering, New Mexico State University, Las Cruces, USA

\begin{abstract}
Biological hydrogels, e.g. bacterial cellulose (BC) hydrogel, attracted increasing interest in recent decades since they show a good potential for biomedical engineering as replacements of real tissues thanks mainly to their good biocompatibility and fibrous structure. To select potential candidates for such applications, a comprehensive understanding of their performance under application-relevant conditions is needed. Most hydrogels demonstrate time-dependent behaviour due to the contribution of their liquid phase and reorientation of fibres in a process of their deformation. To quantify such time-dependent behaviour is crucial due to their exposure to complicated loading conditions in body environment. Some hydrogel-based biomaterials with a multi-layered fibrous structure demonstrate a promise as artificial skin and blood vessels. To
\end{abstract}

\footnotetext{
${ }^{1}$ Corresponding author. Address: Wolfson School of Mechanical and Manufacturing Engineering, Loughborough University, Loughborough Leicestershire LE11 3TU, U.K., Phone: +44/(0)1509/227504, Fax: +44/(0)1509/227502. E-mail: V.Silberschmidt@lboro.ac.uk
} 
characterise and model time-dependent behaviour of these multi-layered hydrogels along their through-thickness direction is thereby of vital importance. Hence, a holistic study combining mechanical testing and micro-morphological observations of BC hydrogel with analytical modelling of its relaxation behaviour based on fraction-exponential operators was performed. The results show a good potential to use a fraction-exponential model to describe such behaviour of multi-layered hydrogels, especially at stages of stress decay at low forces and of stress equilibrium at high forces.

Key words: bacterial cellulose hydrogel; rheology; fraction-exponential operators; stress-relaxation test; time-dependent behaviour

\section{Introduction}

Some biological hydrogels such as bacterial cellulose (BC) hydrogel (Fig. 1a), have been extensively studied mainly thanks to their promise as replacements of real tissue in biomedical engineering [1]. Biologically, they demonstrate good biocompatibility making it possible to provide suitable interactions with adjacent tissues when introduced into body environment [2]. Structurally, hydrogels mostly consist of a fibrous network embedded into interstitial water that mimics architecture of load-bearing components of some real tissues [3]. Mechanically, hydrogels should provide deformational behaviour similar to that of real tissues under relevant conditions [4].

BC hydrogels synthesized by certain groups of bacteria (e.g. Acetobacter, Rhizobium, Agrobacteriumand and Sarcina). High-crystalline long fibres naturally bonded by surrounding water molecules with hydro-bonds are randomly distributed in fibrous layer (Fig. 1b) with a 
small group of cross-links to form multi-layered structure along through-thickness direction (Fig. 1c). Their excellent biological properties and structural features make them a good potential candidate for various applications, including an implant replacement of human tissues (e.g. ear cartilage [5], cornea [6], skins [7], blood vessels [8, 9], etc.) and a use as a scaffold material for both BC-based bio-composites (e.g. BC/fibrin composite for artificial blood vessel [10], BC/polyvinyl alcohol composite for artificial heart-valve leaflets [11], etc.) and in-vitro tissue regeneration (e.g. cortical bone [12], muscle [13, 14], peripheral nerves [15], etc.). Still, the incomplete knowledge of their mechanical characteristics, especially time-dependent behaviour, complicates understanding of their performance under various loading conditions.

Most biomaterials demonstrate time-dependent behaviour mainly thanks to viscous contribution of liquid content; this is true for BC hydrogels. A comprehensive understanding of such behaviour is crucial since they are expected to be exposed to complex loading conditions of body environment [16]. Several experimental studies were performed to characterise viscoelastic properties of biological hydrogels. As an example, stress-relaxation indentation was carried out to study the viscoelastic properties of BC hydrogels with various cellulose contents in order to identify the possibility for ear-cartilage replacement [17]; creep tests were used to investigate time-dependent rheological behaviour at various stress levels [18]. Prediction of mechanical behaviour using an appropriate model is a key for an in-depth understanding of responses to various loading regimes. A classic generalized Maxwell viscoelastic model with a spring/dashpot combination is widely used for this. A modified model combining parallel sets spring/dashpot and a dashpot in series was developed to describe cyclic creep behaviour of a cellulose-based material [19].

A hypothesis to use spring/dashpot combinations in description of viscoelastic behaviour is based 
on assumption that materials are elastic-viscoelastic [20]. The spring and dashpot components describe physical phenomena underpinning viscoelastic behaviour; still, their inconvenience is related to challenges linked to obtaining analytical formulas for an inverse transform in a Fourier or Laplace domain. Consequently, only some specific cases have a desired solution, making this approach not sufficiently flexible. Alternatively, Scott Blair and Coppen [21, 22] and Rabotnov [23] suggested to use fraction-exponential operators to describe viscoelastic behaviour and demonstrated their advantages of sufficient accuracy in description of experimental data of real materials together with convenience of solving analytical Laplace transformations. Thanks to that, the use of fraction-exponential operators in recent years attracted a growing interest in the field of solid mechanics, especially mechanics of heterogeneous materials [24, 25, 26, 27].

Hence, in this paper, a combined approach, including mechanical testing, micro-morphological observations and analytical description with fraction-exponential operators, was used to investigate through-thickness stress relaxation behaviour of a multi-layered BC hydrogel. Our results show that the studied BC hydrogel has typical stress-relaxation behaviour along its through-thickness direction. The extent of layer aggregation dominates this behaviour at increased force levels. The fraction-exponential operators are effective, sensitive and flexible in description of compressive through-thickness stress-relation behaviour of the multi-layered fibrous hydrogel, reflecting a good potential of this technique for analysis of such behaviour at low and high force levels.

\section{Fraction-exponential operators}

A brief introduction into fraction-exponential formulism for description of time-dependent behaviour is given here; for its details can be found elsewhere [18]. To describe viscoelastic 
properties of the material, an integral form of the respective governing equation is used:

$\varepsilon_{i j}(x, t)=S_{i j k l} \sigma_{k l}(x, t)+\int_{0}^{t} K_{i j k l}(t-\tau) \sigma_{k l}(x, \tau) d \tau$,

where $\varepsilon_{i j}$ and $\sigma_{k l}$ are the strain and the stress tensors, respectively, $S_{i j k l}$ is a fourth rank tensor of instantaneous elastic compliance and $K_{i j k l}(t)$ is the time-dependent forth-rank tensor (creep kernel). It is assumed that volume changes during deformation are purely elastic. Then, for an isotropic material, expression (1) can be written as

$\varepsilon_{i j}(x, t)=\frac{1}{3 K_{0}}\left(\frac{1}{3} \delta_{i j}\right) \sigma_{k k}(x, t)+\frac{1}{2}\left(\mu^{*}\right)^{-1}\left(\sigma_{i j}(x, t)-\frac{1}{3} \delta_{i j} \sigma_{k k}(x, t)\right)$,

where

$$
\left(\mu^{*}\right)^{-1}[f(x, t)]=\frac{1}{\mu_{0}} f(x, t)+\int_{0}^{t} J(t-\tau) f(x, \tau) d \tau
$$

where $\mu_{0}$ is instantaneous shear modulus, and $J(t-\tau)$ is the creep kernel. Fraction-exponential functions are used to model this kernel, and details can be found in our previous work [18]. Then, creep and stress relaxation behaviour can be described by fractional-exponential operators:

$$
\begin{aligned}
& \left(\mu^{*}\right)[\varepsilon(x, t)]=\mu_{0}\left[\varepsilon(x, t)+\lambda \int_{0}^{t} \ni_{\alpha}\left(\beta, t-t^{\prime}\right) \varepsilon\left(x, t^{\prime}\right) d t^{\prime}\right], \\
& \left(\mu^{*}\right)^{-1}[\sigma(x, t)]=\frac{1}{\mu_{0}}\left[\sigma(x, t)-\lambda \int_{0}^{t} \ni_{\alpha}\left(\beta-\lambda, t-t^{\prime}\right) \sigma\left(x, t^{\prime}\right) d t^{\prime}\right],
\end{aligned}
$$


where $\alpha, \beta$ and $\lambda$ are material constants, satisfying the following conditions:

$$
\beta<0 ;-1<\alpha \leq 0 ; \beta<\lambda<0 .
$$

\section{Material and method}

\subsection{Synthesis of bacterial cellulose hydrogel}

Gluconacetobacter xylinum (ATCC53582) was used for bio-synthesis of the studied BC hydrogel. The bacterium was cultured in a Hestrin and Schramm (HS) medium, which was composed of 2 wt. $\%$ glucose, 0.5 wt.\% yeast extract, 0.5 wt.\% peptone, 0.27 wt.\% disodium phosphate, and 0.15 wt.\% citric acid. After incubating statically for 7 days at $30^{\circ} \mathrm{C}$, resulting in thickness of the $\mathrm{BC}$ hydrogel in the range approximately from $3 \mathrm{~mm}$ to $5 \mathrm{~mm}$, the $\mathrm{BC}$ hydrogel was dipped into deionized (DI) water for 2 days, and then steamed by boiling in a $1 \mathrm{wt} . \% \mathrm{NaOH}$ solution for 30 mins to eliminate bacteria and proteins. Afterwards, samples of the BC hydrogel (Fig. 1a) were purified by washing in DI water until their $\mathrm{pH}$ value approached 7, and then were stored in DI water at $4^{\circ} \mathrm{C}$.

\subsection{Compressive stress-relaxation tests}

Cylinder-shape specimens for compressive stress-relaxation tests were cut with a standard hollow punch with inner diameter of $20 \mathrm{~mm}$ (Fig. 2a). Height of specimens was measured with a calliper with an accuracy of $0.01 \mathrm{~mm}$. To emulate in-vivo conditions, a BioPuls system (Instron 3130-100 BioPuls Bath, Instron, USA) was used with a universal testing machine (Instron 3366, Instron, USA) to provide aqueous testing environment with constant temperature of $37.0 \pm 1.0^{\circ} \mathrm{C}$ 
(Figs. 2b-c). A pre-load of $0.05 \mathrm{~N}$ was used to minimize the effect of flotage. Specimens $(n=5)$ were first compressed to force levels of $25 \mathrm{~N}, 50 \mathrm{~N}, 100 \mathrm{~N}$ and $200 \mathrm{~N}$, and then the achieved level of displacement was hold for 1.5 hrs. Magnitudes of force and displacement were measured with a 500 N load cell (2530 Series Low-profile Static Load Cell, Instron, USA) and a crosshead displacement sensor, respectively, with data-acquisition frequency of $1 \mathrm{~Hz}$.

\subsection{Micro-morphological observations}

Microstructure of the studied BC hydrogel at various force levels is vital to understand its stress-relaxation behaviour. In this study, cylinder-shape specimens were first compressed to force levels of $1 \mathrm{~N}, 25 \mathrm{~N}$ and $200 \mathrm{~N}$. Then, water in compressed specimens was removed in a freeze-dryer in order to maintain the structure. The resulting fibrous structure was observed with field emission-gun scanning electron microscopy.

\subsection{Assessment of fraction-exponential parameters}

An algorithm developed in MATLAB allowed us to fit the experimental data of stress relaxation with the fraction-exponential formulism. The implementation of Mittag-Leffler function available in MATLAB CENTRAL was used to approximate a stress-strain relation,

$$
\sigma(t)=\varepsilon_{0} \mu_{0}\left\{1+\frac{\lambda}{\beta}\left[\mathrm{M}_{\alpha+1}\left(\beta t^{\alpha+1}\right)-1\right]\right\} .
$$

The magnitude of $\mu_{0}$ was found as $\mu_{0}=\sigma(0)$. Values of the remaining parameters $-\lambda, \alpha$ and $\beta$ - were approximated with a nonlinear least-square method. In our case, this was performed using a MATLAB lsqnonlin function with initial guesses of $-0.5,-0.1$, and 0.0005 , respectively. The accuracy of approximation was calculated with Resnorm $R$ that is 
$l^{2}$-norm of fit to present the accuracy of curve fitting, defined as

$$
R=\sum\left[\sigma_{E}(t)-\sigma_{F}(t)\right]^{2},
$$

where $\sigma_{E}(t)$ is the experimental strain data, $\sigma_{F}(t)$ is the derived function.

\section{Results and Discussion}

Through-thickness compressive stress-relaxation (stress-time) curves for the studied BC hydrogel are shown in Fig. 3 for four force levels (pre-relaxation domains are omitted). The hydrogel demonstrates typical stress-relaxation behaviour; its stress decay rate is extremely high at the beginning of relaxation for each force level. As a result, the specimens for all the force levels demonstrated a stress-relaxation time of less than 100 s; an inset in Fig. 3 presents the behaviour for a time period from 0 to $300 \mathrm{~s}$. The time required for full relaxation (when stress approaches equilibrium) grows with the increased initial force. At each force level, the stress level declined by over $80 \%$ before reaching a static state. Because of the free-water content, the high level of stress decay could be the result of water movement.

The lack of knowledge of structure-function relationships is mainly due to a challenge in observations of microstructural changes under deformation, especially for nano-fibrous systems in aqueous environment. To achieve understanding of mechanisms underpinning compressive stress relaxation, an additional study of compression of the BC hydrogel was performed; also morphology of its layered structure along through-thickness direction was obtained for force levels of $1 \mathrm{~N}, 25 \mathrm{~N}$ and $200 \mathrm{~N}$. 
A typical through-thickness compressive behaviour of the BC hydrogel is presented in Fig. 4a. Beyond the force level of $25 \mathrm{~N}$, stress increased with an extremely high rate. From micro-morphological observations, the cross-sectional structure of fibrous layers can be easily recognized at force level of $1 \mathrm{~N}$ (Fig. 4b). By increasing compressive loading to $25 \mathrm{~N}$, some fibrous layers were compacted closely while others were not, demonstrating non-uniformity of a through-thickness compaction process (Fig. 4c). When the compressive load continuously increased to $200 \mathrm{~N}$, most layers were fully compacted (Fig. 4d).

The compressive results with micro-morphological observations suggested a process of layer aggregation. Compressive load attempted to press water out and compact layers. During the stage of force increasing from 0 to $25 \mathrm{~N}$, compressive deformation changed swiftly since fibrous layers pressed free water out, and the behaviour was mostly defined by the flow process.

When the force reached $25 \mathrm{~N}$, a drastic stiffening process occurred resulting from the aggregation of fibrous layers (Fig. 4c-d). In stress-relaxation, all the studied force levels (25 $200 \mathrm{~N}$ ) are located in this region; hence, it is assumed that performance of fibrous layers dominates the studied stress-relaxation behaviour rather than the process of squeezing water out of the specimens.

The obtained experimental results were used to develop the fraction-exponential model of hydrogel's viscosity. Since the BC hydrogel shows potential replacement of blood vessels, skin, etc., a description of its compressive stress-relaxation behaviour with good accuracy is desirable. Based on the fractional-exponential formulism, the developed numerical procedure provided the best solution for each force levels with Resnorm values less than 0.0001 . The fractional-exponential operators are sensitive to description of stress-relaxation behaviour of the 
BC hydrogel at various force levels, showing excellent agreement with the experimental results (Fig. 5). The obtained fraction-exponential parameters with respective Resnorm values (fit accuracy) are summarized in Tab. 1.

In fraction-exponential operators, parameter $\mu_{0}$ represents the instantaneous shear modulus; it increased with a growing force level indicating a material-stiffening process. As a result of layer aggregation, the material becomes stiffer since more fibres contribute to load-bearing, reflected in the observed tendency of $\mu_{0}$. Parameters $\lambda, \alpha$ and $\beta$ for all the studied force levels satisfied the basic requirements of the fraction-exponential operators $(-1<\alpha \leq 0, \beta<\lambda<0)$; still, each set of them differs from those for other force levels. The absolute values of $\lambda$ and $\beta$ decreased with increased force levels, and no clear tendency was found in the evolution of $\alpha$.

Though results in Fig. 5 show a good potential to use the fraction-exponential operators to describe through-thickness stress-relaxation behaviour of the BC hydrogel, the use of a single set of parameters is excepted and more applicable. Due to observed material stiffening, values of $\mu_{0}$ in Tab. 1 reflect the behaviour of the real material. Hence, a single set of $\lambda, \alpha$ and $\beta$ with the force-dependent values of $\mu_{0}$ (as in Tab. 1) was defined, providing the best fit for all the studied force levels (Fig. 6). The obtained magnitudes of parameters with Resonrm values are summarized in Tab. 2.

At low forces $-25 \mathrm{~N}$ and $50 \mathrm{~N}$, the model did not show good agreement at the stages of stress decay and stress equilibrium. The potential reason for this is that, at such loading regimes, not all the fibrous layers were fully compacted, and thereby, there was still some free water moving free and reducing potential energy of the entire system. As a result, the resulting stress relaxation time 
was relatively short. With the increased force level, the fraction-exponential model showed a growing accuracy in description of stress relaxation when it approached equilibrium, indicating that the stress applied to the solid phase of fibres contributed more to the material's response than water movement. At the force level of $100 \mathrm{~N}$, a fitting curve almost coincided with the experimental one after $150 \mathrm{~s}$, reflecting that beyond the force level of $100 \mathrm{~N}$, almost all the fibrous layers were compacted. At the highest force level of $200 \mathrm{~N}$, the model achieved an almost perfect description of through-thickness stress-relaxation behaviour. So, with the increased force level, the effect of water motion reduced until becoming effectively negligible.

Hence, it is suggested that compressive stress relaxation behaviour of multi-layered hydrogels is dominated by water movement and layer aggregation at low- and high-force levels, respectively; thereby, it is reasonable to study such behaviours separately at low-force (25 N and $50 \mathrm{~N}$, in our case) and high-force (100 N and $200 \mathrm{~N}$ ) levels. As shown in Fig. 7, implementation o0f this approach with the fraction-exponential model shows good agreement with observed stress-relaxation behaviours, especially at stage of stress decay at low-force level (Figs. 7a-b) and at stage of stress equilibrium at high-force level (Figs. 7c-d). The respective parameters are summarized in Tab. 3. At low-force level, the model can accurately describe a process when water movement reduces the potential energy of the whole system; while, at high-force level, it perfectly reflects a quasi-static state when fibre-layers bear most stress.

\section{Conclusions}

Through-thickness compressive behaviour of multi-layered hydrogels is of vital importance for real-life biomedical applications. This study experimentally determined time-dependent 
behaviour of the BC hydrogel at various force levels by means of compressive stress-relaxation tests. The use of an in-aqua testing system provided a more realistic application-related environment. Micro-morphological observations allowed identification of structure-function relationships, reflecting two mechanisms - water movement and layer aggregation. The experimentally determined results were accompanied with the use of a fraction-exponential formalism to analytically model the studied behaviour. The fraction-exponential operators are sensitive and flexible to describe time-dependent behaviour providing advantages in solving boundary-value problems for viscoelastic materials and for finite-element simulations of specific geometries and cases. The obtained results showed a good potential of the fraction-exponential operators to describe through-thickness stress-relaxation behaviour of multi-layered hydrogels, especially at stage of stress decay at low-force level and at stage of stress equilibrium at high-force level.

\section{Acknowledgement}

The partial support from the following grants is gratefully acknowledged: FP7 IRSES project TAMER (Grant No. IRSES-GA-2013-610547) (XG, PK, IS and VVS); China-European Union technology cooperation programme (Grant No. 1110) (ZS and GY); M6 Project (Grant No. PIRSES-GA-2010-269113) (CL and VVS).

\section{References}

[1] Shi, Z., Zhang, Y., Phillips, G. O., Yang, G., "Utilization of bacterial cellulose in food,” 
Food Hydrocolloids, vol. 35, pp. 539-545, 2014.

[2] Helenius, G., Backdahl, H., Bodin, A., Nannmark, U., Gatenholm, P., Risberg, B., “In vivo biocompatibility of bacterial cellulose,” J. Biomed. Mater. Res. A, vol. 76A, p. 431-438, 2006.

[3] Koh, C.T., Strange, D.G.T., Tonsomboon, K., Oyen, M.L., "Failure mechanisms in fibrous scaffolds,” Acta Biomater., vol. 9, p. 7326-7334, 2013.

[4] Gao, X., Shi, Z., Liu, C., Yang, G., Sevostianov, I., Silberschmidt, V.V., "Inelastic behaviour of bacterial cellulose hydrogel:In aqua cyclic tests,” Polym. Test., vol. 44, pp. 82-92, 2015.

[5] Nimeskern, L., Avila, H.M., Sundberg, J., Gatenholm, P., Muller, R., Stok, K.S., “Mechanical evaluation of bacterial nanocellulose as an implant material for ear cartilage replacement,” J. Mech. Behav. Biomed. Mater., vol. 22, pp. 12-21, 2013.

[6] Wang, J., Gao, C., Zhang, Y., Wan, Y., "Preparation and in vitro characterization of BC/PVA hydrogel composite for its potential use as artificial cornea biomaterial,” Mater. Sci. Eng. C, vol. 30, pp. 214-218, 2010.

[7] Gibas, I., Janik, H. , “Review: synthetic polymer hydrogels for biomedical applications,” Ch\&ChT., vol. 4, no. 4, pp. 297-304, 2010.

[8] Malho, J., Laaksonen, P., Walther, A., Ikkala, O., Linder, M.B., "Facile Method for Stiff, Tough, and Strong Nanocomposites by Direct Exfoliation of Multilayered Graphene into Native Nanocellulose Matrix,” Biomacromolecules, vol. 13, p. 1093-1099, 2012.

[9] Zang, S., Zhang, R., Chen, H., Lu, Y., Zhou, J., Chang, X., Liu, G., Wu, Z., Yang, G., “Investigation on artificial blood vessels prepared from bacterial cellulose,” Mater. Sci. Eng. C, vol. 46, pp. 111-117, 2015. 
[10] Brown, E.E., Zhang, J., Laborie , M.G., “Never-dried bacterial cellulose/fibrin composites: preparation, morphology and mechanical properties,” Cellulose, vol. 18, pp. 631-641 , 2011.

[11] Millon, L.E., Wan, W.K., "The polyvinyl alcohol-bacterial cellulose system as a new nanocomposite for biomedical applications,” Biomed. Mater. Res. B, vol. 79, pp. 245-253, 2006.

[12] Zaborowska, M., Bodin, A., Bäckdahl, H., Popp, J., Goldstein, A., Gatenholm, P., “Microporous bacterial cellulose as a potential scaffold for bone regeneration,” Acta Biom., vol. 6, pp. 2540-2547, 2010.

[13] Bäckdahl, H., Helenius, G., Bodin, A., Nannmark, U., Johansson, B. R., Risberg, B., Gatenholm, P., "Mechanical properties of bacterial cellulose and interactions with smooth muscle cells,” Biomaterials, vol. 27, no. 9, pp. 2141-2149, 2006.

[14] Bäckdahl, H., Esguerra, M., Delbro, D., Risberg, B., Gatenholm, P., "Engineering microporosity in bacterial cellulose scaffolds," J Tissue Eng. Regen. Med. , vol. 2, no. 6, pp. 320-330, 2008.

[15] Kowalska-Ludwicka, K., Grobelski, B., Cala, J., Grobelski, B., Sygut, D., Jesionek-Kupnicka, D., Kolodziejczyk, M., Bielecki, S., Pasieka, Z., “Special paper-New methods Modified bacterial cellulose tubes for regeneration of damaged peripheral nerves,” Arch. Med. Sci., vol. 9, no. 3, pp. 527-534, 2013.

[16] Schiavi, A., Cuccaro, R., Troia, A., "Strain-rate and temperature dependent material properties of Agar and Gellan Gum used in biomedical applications,” J. Mech. Behav. Biomed. Mater, vol. 53, pp. 119-130, 2016.

[17] Nimeskern, L., Avila, H.M., Sundberg, J., Gatenholm, P., Muller, R. and Stok, K.S., 
"Mechanical evaluation of bacterial nanocellulose as an implant material for ear cartilage replacement,” J. Mech. Behav. Biomed. Mater., vol. 22, pp. 12-21, 2013.

[18] Gao, X., Shi, Z., Kuśmierczyk, P., Liu, C., Yang, G., Sevostianov, I., Silberschmidt, V.V., “Time-dependent rheological behaviour of bacterial cellulose hydrogel,” Mater. Sci. Eng. C, vol. 58, pp. 153-159, 2016.

[19] Kim, J., Ampofo, J., Craft, W., Kim, H.S., "Modeling elastic, viscous, and creep characteristics of cellulose Electro-Active Paper,” Mech. Mater., vol. 40, pp. 1001-1011, 2008.

[20] Christensen, R.M., Theory of Viscoelasticity, Academic Press, 1982.

[21] Scott Blair, G.W., Coppen, F.M.V., "The estimation of firmness in soft materials,” Amer. J. Psychol., vol. 56, pp. 234-246, 1943.

[22] Scott Blair, G.W., Coppen, F.M.V., "The subjective judgement of the elastic and plastic properties of soft bodies: the differential thresholds for viscosities and compression moduli,” Proc. Roy. Soc. B, vol. 128, pp. 109-125, 1939.

[23] Rabotnov, Yu.N., "Equilibrium of an elastic medium with after-effects (in Russian).," $J$. Appl. Math. Mech., vol. 12, pp. 53-62, 1948; reprinted in Engl. in: Fract. Calc. Appl. Anal. 17, No 3 (2014), 684-696; DOI: 10.2478/s13540-014-0193-1.

[24] Di Paola, M., Zingales, M., “Exact mechanical models of fractional hereditary materials,” $J$. Rheol., vol. 56, pp. 983-1004, 2012.

[25] Levin, V., Sevostianov, I., "Micromechanical modeling of the effectiveviscoelastic properties of inhomogeneous materials using fraction-exponential operators," Int. J. Fracture, vol. 134, pp. L37-L44, 2005. 
[26] Sevostianov, I., Levin, V., “Creep and relaxation contribution tensors for spheroidal pores in hereditary solids: fraction-exponential operators approach,” Acta Mech., pp. DOI 10.1007/s00707-015-1426-z, 2015.

[27] Sevostianov, I., Levin, V.M., Radi, E., "Effective properties of linear viscoelastic microcracked materials: application of Maxwell homogenization scheme,” Mech. Mater., vol. 84, pp. 28-43, 2015. 


\section{Figure captions}

Figure 1: (a) BC hydrogel. SEM images of the studied BC hydrogel show randomly distributed fibres in a fibrous layer (b) (radial-transverse plane) and a multiple-layer structure (c) with some links between layers along the through-thickness direction. (d) Schematic diagram of radial, transverse and through-thickness directions.

Figure 2: (a) Cylinder-shape specimen for compressive stress relaxation cut with standard hollow punch; (b) experimental set-up with BioPuls system providing in-aqua testing conditions (c)

Figure 3: Obtained experimental data show typical stress-relaxation behaviour of BC hydrogel at various force levels

Figure 4: (a) Typical through-thickness compressive behaviour of BC hydrogel with corresponding cross-sectional microstructures at force level of $1 \mathrm{~N}$ (b), $25 \mathrm{~N}$ (c) and $200 \mathrm{~N}$ (d)

Figure 5: A fraction-exponential model is sensitive enough to describe stress-relaxation behaviour of the BC hydrogel. The numerical fit curves with various parameters show perfect agreement with experimental ones at force levels of $25 \mathrm{~N}$ (a), $50 \mathrm{~N}$ (b), $100 \mathrm{~N}$ (c) and $200 \mathrm{~N}$ (d).

Figure 6: A fraction-exponential model with various magnitudes of $\boldsymbol{\mu}_{\mathbf{0}}$ and constant magnitudes of $\boldsymbol{\lambda}, \boldsymbol{\alpha}$ and $\boldsymbol{\beta}$ at force levels of $25 \mathrm{~N}$ (a), $50 \mathrm{~N}$ (b), $100 \mathrm{~N}$ (c) and $200 \mathrm{~N}$ (d) reflects dissimilarity of stress relaxation behaviour at low-force (25 N and $50 \mathrm{~N}$ ) and high-force (100 N and $200 \mathrm{~N}$ ) levels.

Figure 7: A fraction-exponential model with various $\boldsymbol{\mu}_{\mathbf{0}}$, and two sets of constant $\boldsymbol{\lambda}$, $\boldsymbol{\alpha}$ and $\boldsymbol{\beta}$ for (i) low-force level of $25 \mathrm{~N}$ (a) and $50 \mathrm{~N}$ (b) and (ii) high-force level of $100 \mathrm{~N}$ (c) and $200 \mathrm{~N}$ (d) indicates its suitability for description of stress-relaxation behaviour of the BC hydrogel at different force levels. 


\section{Table captions}

Table 1: Determined fraction-exponential parameters of various $\boldsymbol{\mu}_{\mathbf{0}}, \boldsymbol{\lambda}, \boldsymbol{\alpha}$ and $\boldsymbol{\beta}$, and Resnorm $R$ for each force level

Table 2: Determined fraction-exponential parameters of various $\boldsymbol{\mu}_{\mathbf{0}}$, and constant $\boldsymbol{\lambda}, \boldsymbol{\alpha}$ and $\boldsymbol{\beta}$, and Resnorm $R$ for each force levels

Table 3: Determined fraction-exponential parameters of various $\boldsymbol{\mu}_{\mathbf{0}}$, and constant $\boldsymbol{\lambda}$, $\boldsymbol{\alpha}$ and $\boldsymbol{\beta}$, and Resnorm R for two ranges of force: low forces (25 N and $50 \mathrm{~N}$ ) and high forces (100 N and $200 \mathrm{~N})$ 


\section{Graphic abstract}

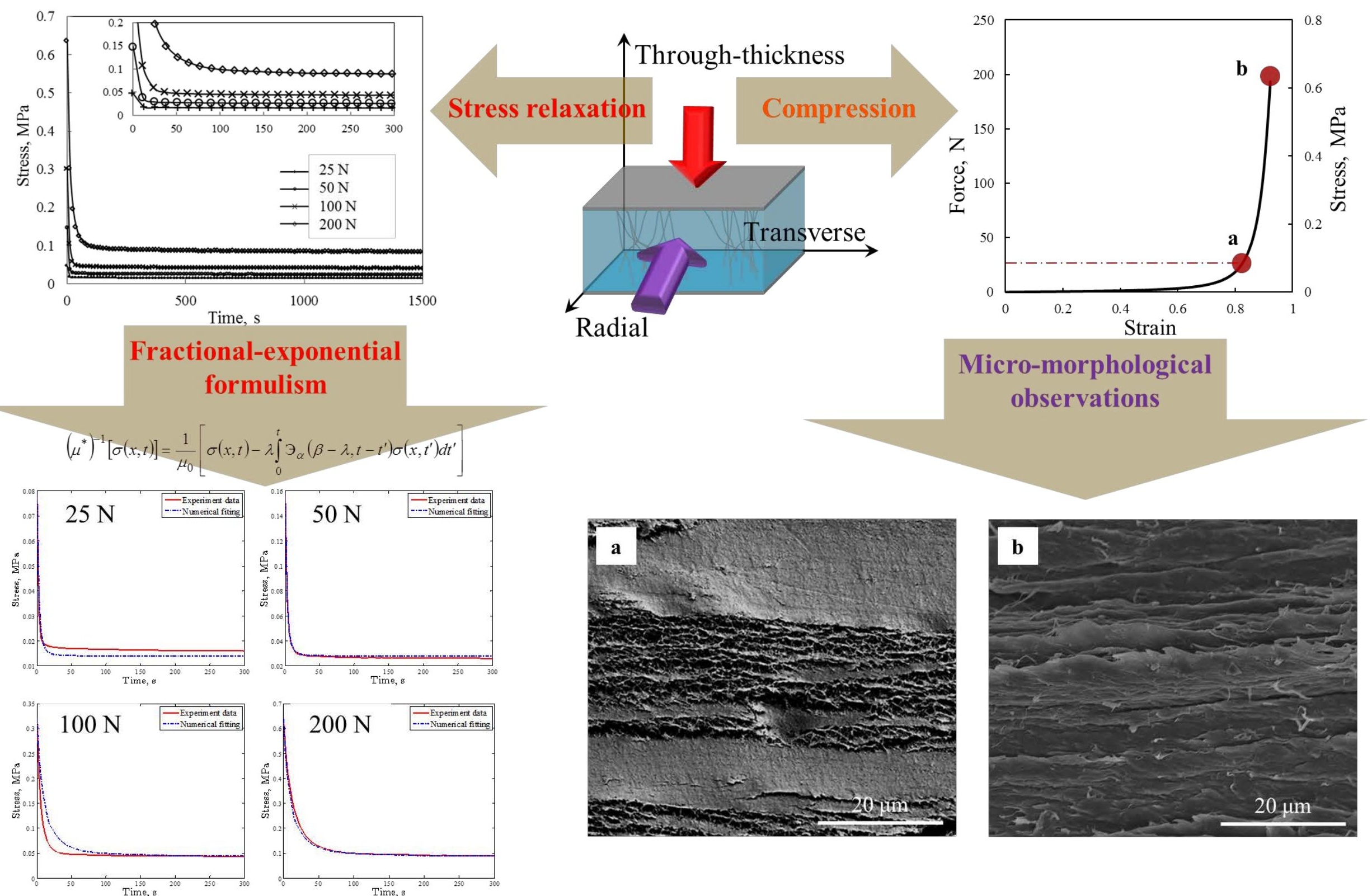


Figure 1: (a) BC hydrogel. SEM images of the $B C$ hydrogel show randomly distributed fibres in a fibrous layer (b) (radial-transverse plane), and a multiple-layer structure (c) with some links between layers along the out-of-plane direction. (d) Schematic diagram of radial, transverse and out-of-plane directions.
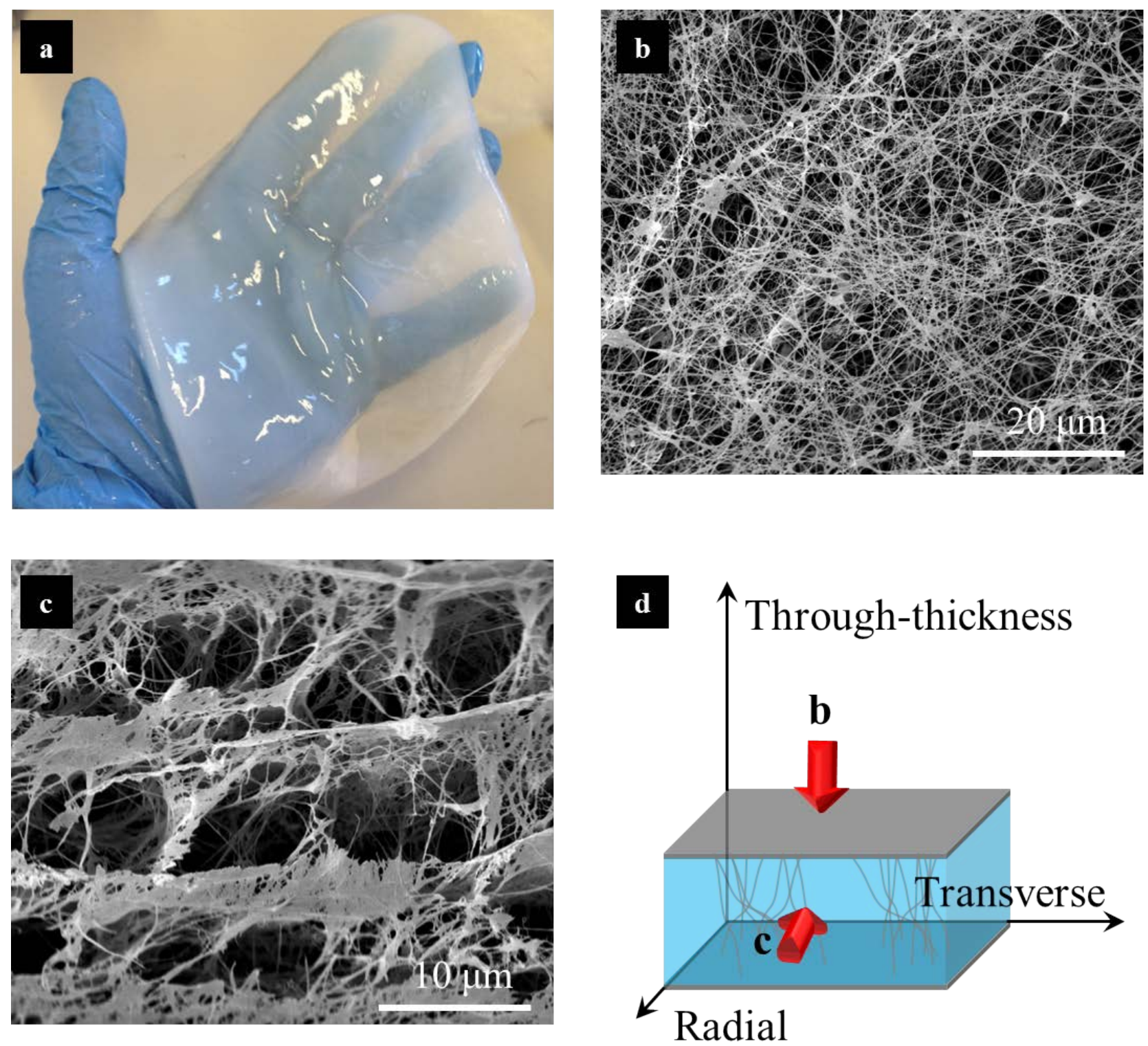
Figure 2: (a) Cylinder-shape specimens (top) for compressive stress relaxation cut with standard hollow punch (bottom); (b) experimental set-up with BioPuls system providing in-aqua testing conditions (c)
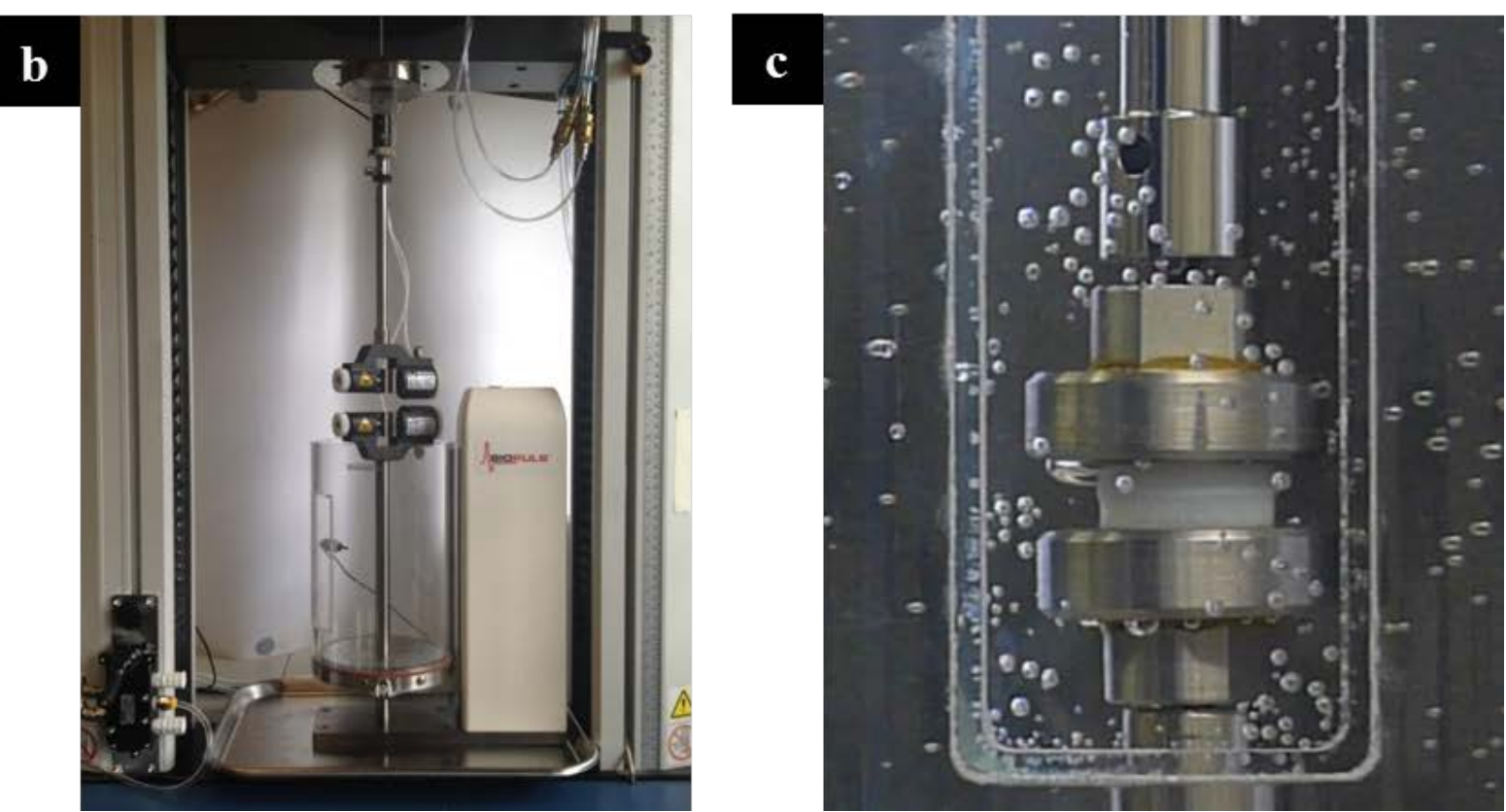
Figure 3: Obtained experimental data shows typical stress-relaxation behaviour of $B C$ hydrogel at various force levels

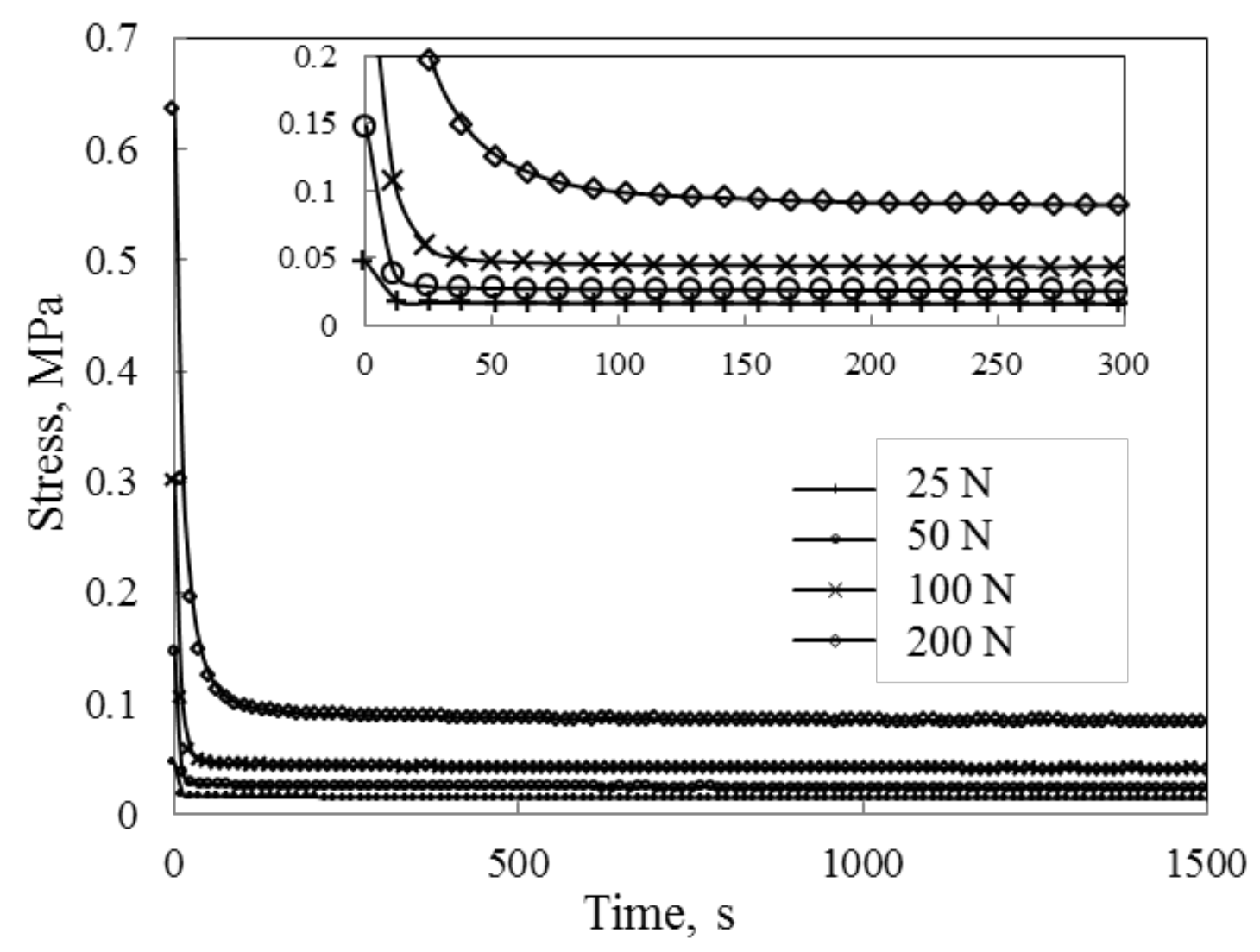


Figure 4: (a) Typical out-of-plane compressive behaviour of BC hydrogel with corresponding cross-sectional microstructures at force level of $1 \mathrm{~N}$ (b), $25 \mathrm{~N}$ (c) and $200 \mathrm{~N}$ (d)
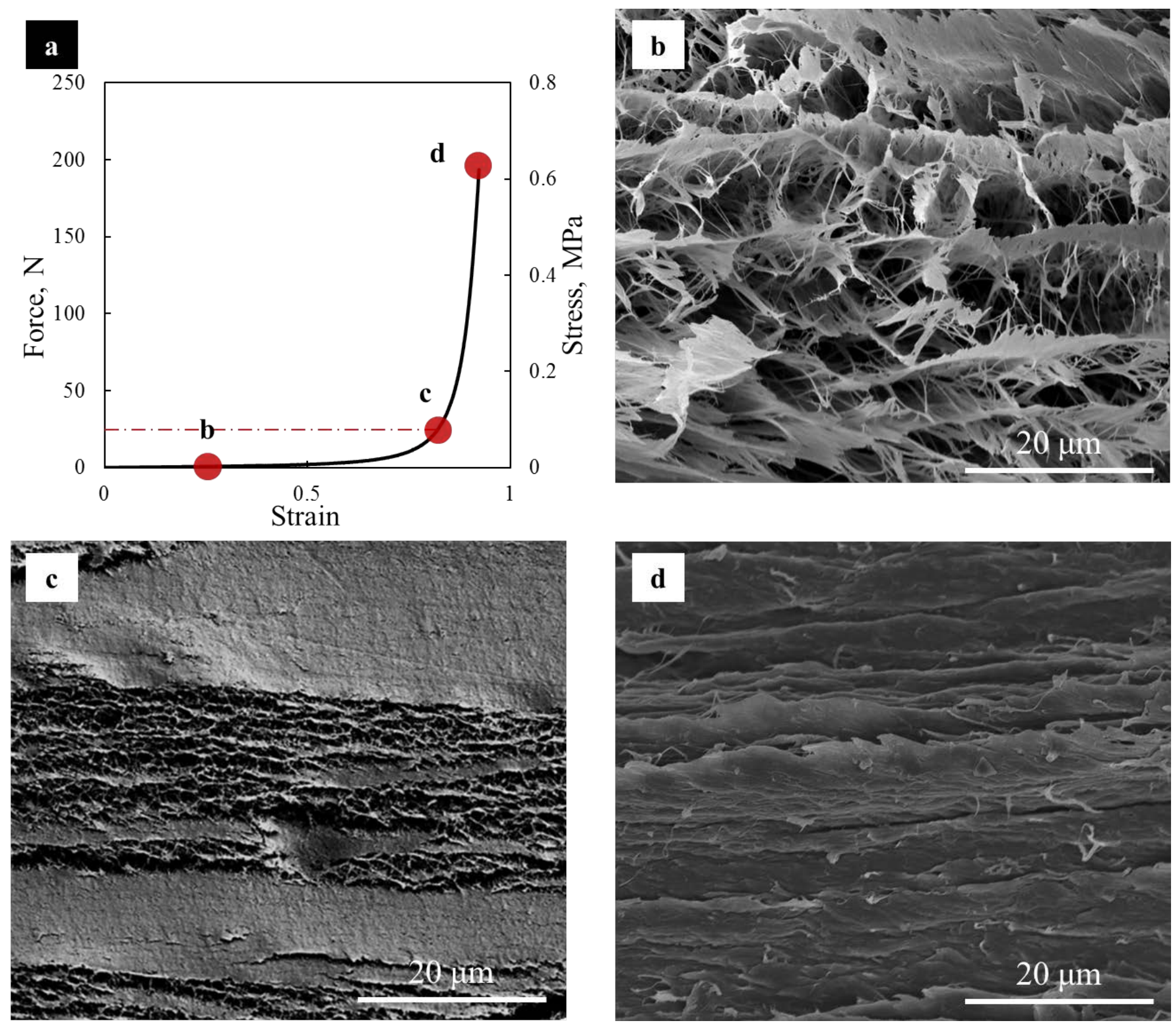
Figure 5: Fraction-exponential model is sensitive enough to describe the stress relaxation behaviour of $\mathrm{BC}$ hydrogel. The numerical fit curves with various parameters show perfect agreement with experimental ones at force levels of $25 \mathrm{~N}(\mathrm{a}), 50 \mathrm{~N}(\mathrm{~b}), 100 \mathrm{~N}$ (c) and $200 \mathrm{~N}$ (d).
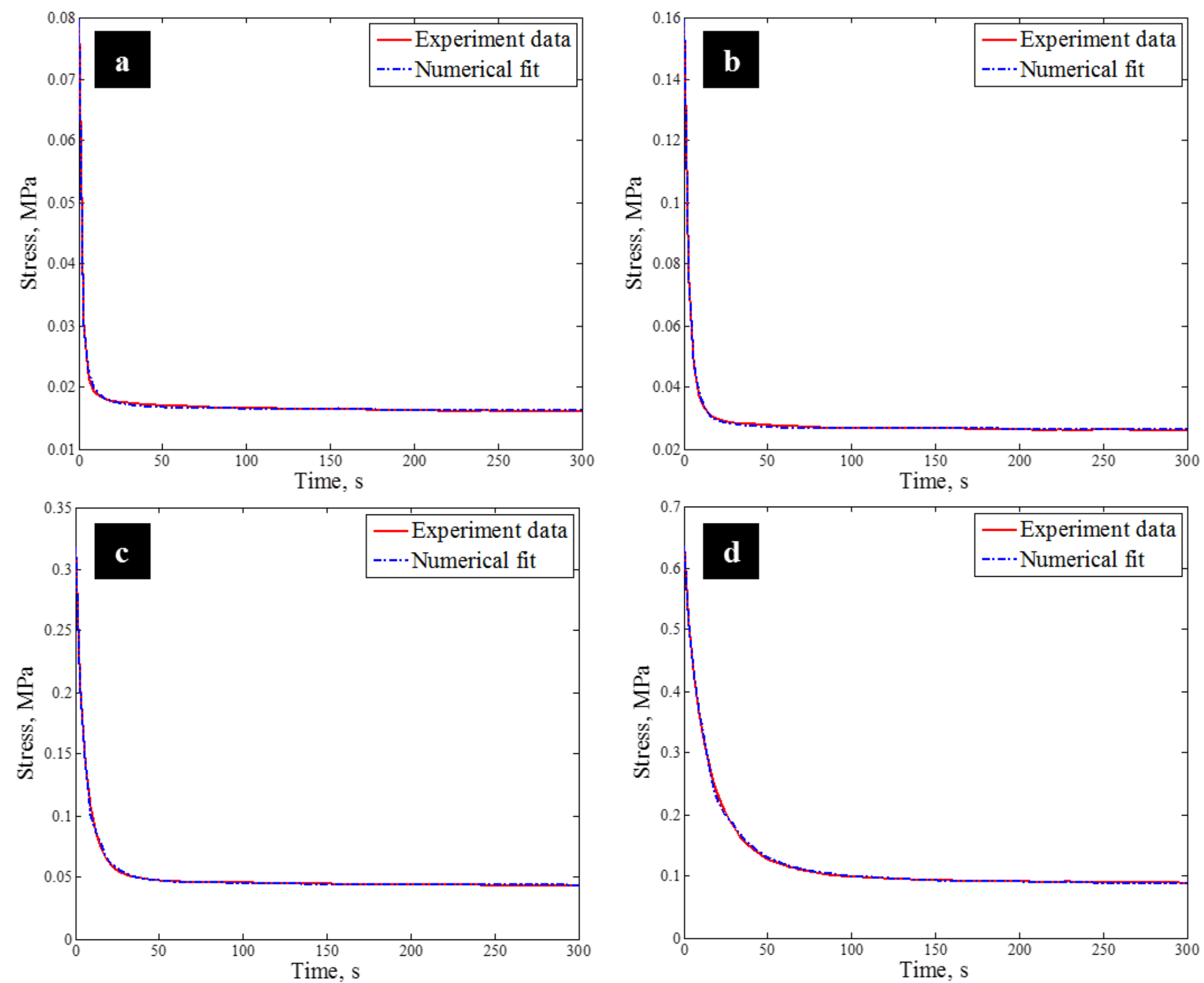
Figure 6: Fraction-exponential model with various $\mu_{0}$, , and constant $\lambda, \alpha$ and $\beta$, show sufficient accuracy with the experimental ones at force levels of $25 \mathrm{~N}(\mathrm{a}), 50 \mathrm{~N}$ (b), $100 \mathrm{~N}$ (c) and $200 \mathrm{~N}$ (d)., indicating the possibility to use fraction-exponential model to describe stress-relaxation behaviour of BC hydrogel.
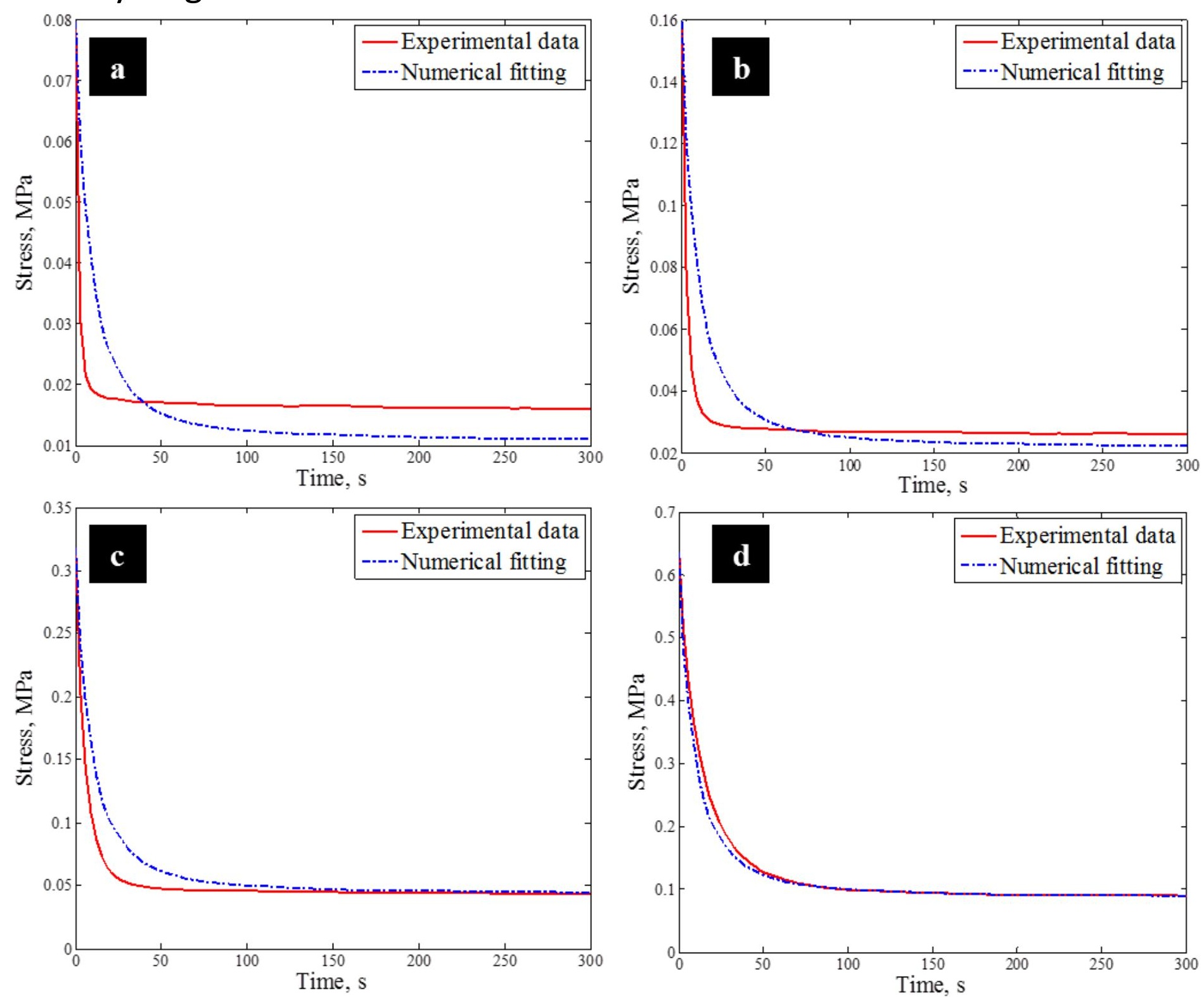
Figure 7: Fraction-exponential model with various $\mu_{0}$, and constant $\lambda, \alpha$ and $\beta$ for low-force level of $25 \mathrm{~N}$ (a) and $50 \mathrm{~N}$ (b), and high-force level of $100 \mathrm{~N}$ (c) and $200 \mathrm{~N}$ (d) indicates the possibility to use fraction-exponential model to describe stress-relaxation behaviour of the $\mathrm{BC}$ hydrogel at low and high force levels.
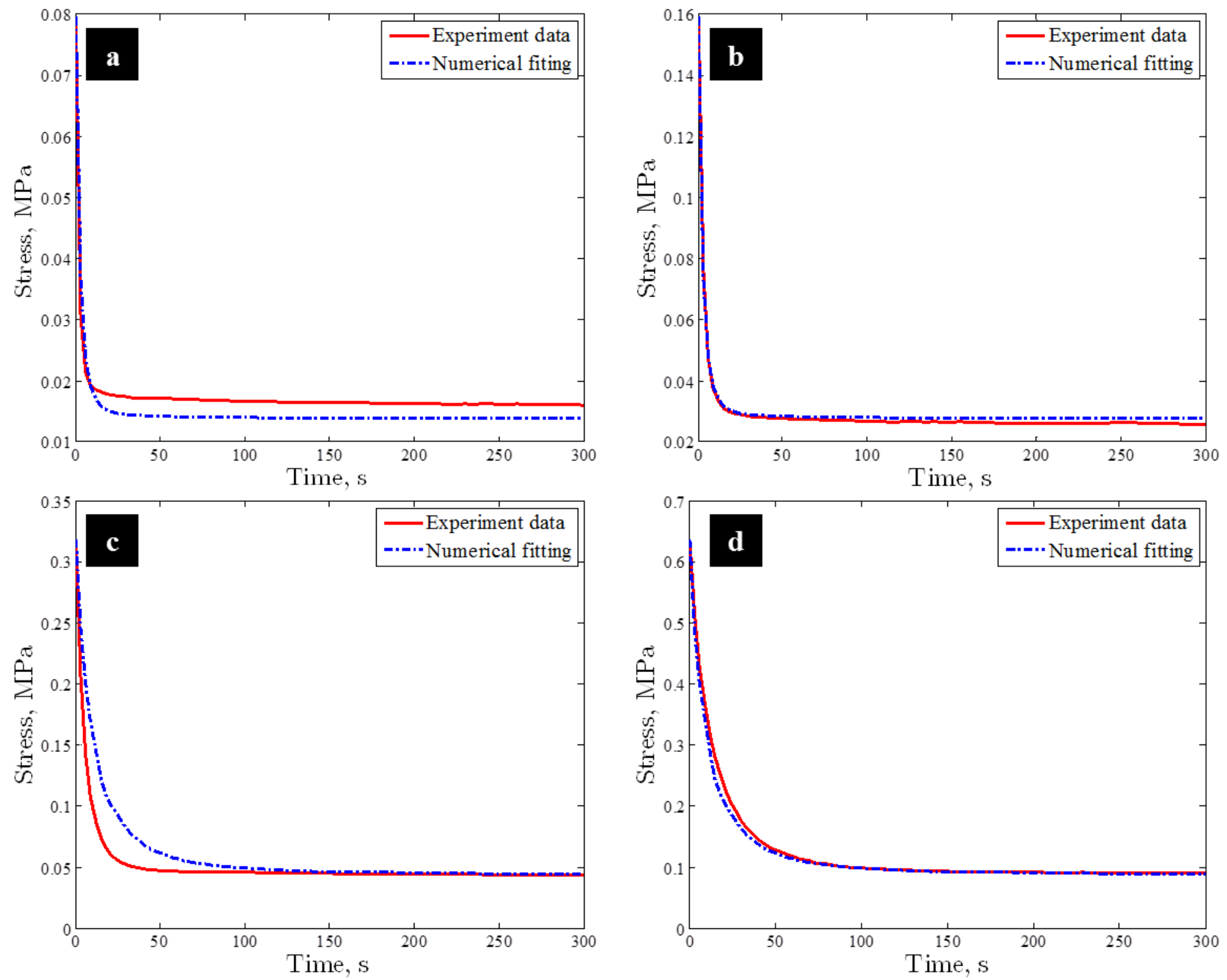
Table 1: Determined fraction-exponential parameters of various $\mu_{0}, \lambda, \alpha$ and $\beta$, and Resnorm $R$ for each force levels

\begin{tabular}{cccccc}
\hline Force level, $\mathbf{N}$ & $\boldsymbol{\mu}_{\mathbf{0}}$ & $\boldsymbol{\lambda}$ & $\boldsymbol{\alpha}$ & $\boldsymbol{\beta}$ & $\begin{array}{c}\boldsymbol{R} \\
\text { (Fitting accuracy) }\end{array}$ \\
\hline $\mathbf{2 5}$ & 0.08 & $-5.15 \times 10^{-1}$ & $-1.49 \times 10^{-1}$ & $-6.48 \times 10^{-1}$ & $4.32 \times 10^{-6}$ \\
$\mathbf{5 0}$ & 0.16 & $-3.00 \times 10^{-1}$ & $-7.71 \times 10^{-2}$ & $-3.59 \times 10^{-1}$ & $9.61 \times 10^{-6}$ \\
$\mathbf{1 0 0}$ & 0.30 & $-1.65 \times 10^{-1}$ & $-7.36 \times 10^{-2}$ & $-1.91 \times 10^{-1}$ & $7.35 \times 10^{-5}$ \\
$\mathbf{2 0 0}$ & 0.59 & $-8.10 \times 10^{-2}$ & $-1.10 \times 10^{-1}$ & $-9.32 \times 10^{-2}$ & $5.23 \times 10^{-4}$ \\
\hline
\end{tabular}


Table 2: Determined fraction-exponential parameters of various $\mu_{0}, \lambda, \alpha$ and $\beta$, and Resnorm $R$ for each force levels

\begin{tabular}{cccccc}
\hline Force level, $\mathbf{N}$ & $\boldsymbol{\mu}_{\mathbf{0}}$ & $\boldsymbol{\lambda}$ & $\boldsymbol{\alpha}$ & $\boldsymbol{\beta}$ & $\begin{array}{c}\boldsymbol{R} \\
\text { (Fitting accuracy) }\end{array}$ \\
\hline $\mathbf{2 5}$ & 0.08 & & & $2.19 \times 10^{-2}$ \\
$\mathbf{5 0}$ & 0.16 & $-1.07 \times 10^{-1}$ & $-1.03 \times 10^{-1}$ & $-1.22 \times 10^{-1}$ & $1.12 \times 10^{-2}$ \\
$\mathbf{1 0 0}$ & 0.30 & & & $1.10 \times 10^{-2}$ \\
$\mathbf{2 0 0}$ & 0.59 & & & $4.36 \times 10^{-3}$ \\
\hline
\end{tabular}


Table 3: Determined fraction-exponential parameters of various $\mu_{0}$, and constant $\lambda, \alpha$ and $\beta$, and Resnorm R for low-force (25 N and $50 \mathrm{~N}$ ) and high-force (100 N and $200 \mathrm{~N}$ ) levels

\begin{tabular}{cccccc}
\hline Force level, $\mathbf{N}$ & $\boldsymbol{\mu}_{\mathbf{0}}$ & $\boldsymbol{\lambda}$ & $\boldsymbol{\alpha}$ & $\boldsymbol{\beta}$ & $\begin{array}{c}\boldsymbol{R} \\
\text { (Fitting accuracy) }\end{array}$ \\
\hline $\mathbf{2 5}$ & 0.08 & $-3.02 \times 10^{-1}$ & $-6.91 \times 10^{-2}$ & $-3.65 \times 10^{-1}$ & $6.46 \times 10^{-4}$ \\
$\mathbf{5 0}$ & 0.16 & & & $1.66 \times 10^{-4}$ \\
$\mathbf{1 0 0}$ & 0.30 & $-9.81 \times 10^{-2}$ & $-1.24 \times 10^{-1}$ & $-1.91 \times 10^{-1}$ & $2.52 \times 10^{-2}$ \\
$\mathbf{2 0 0}$ & 0.59 & & & & $6.84 \times 10^{-3}$ \\
\hline
\end{tabular}

